\title{
ESTUDIOS CITOGENÉTICOS EN HÍBRIDOS TRIPLOIDES Y TETRAPLOIDES DE TURNERA KRAPOVICKASII, T. SCABRA Y T. SUBULATA (PASsifloraceae, Turneroideae)
}

\author{
AVELIANO FERNÁNDEZ ${ }^{1}$, SILVIA A. FERNÁNDEZ ${ }^{1 *}$, IVANA E. KOVALSKY ${ }^{1,2}$ y \\ VIVIANA G. SOLÍS NEFFA ${ }^{1,2}$
}

\begin{abstract}
Summary: Cytogenetic studies in triploid and tetraploid hybrids of Turnera krapovickasii, T. scabra and T. subulata (Passifloraceae, Turneroideae). In order to investigate the genomic relationships among species of series Turnera $(x=5)$, a controlled crossing program was performed and yielded numerous hybrids. Among the yellow flowered species of series Turnera, T. krapovickasii, T. scabra and T. subulata have diploid and autotetraploid cytotypes. So far, the degree of reproductive isolation between diploids and autotetraploids, and the degree of genomic affinity at tetraploid level are unknown. In this study, we present the results of the cytogenetic analysis of triploid $(2 n=3 x=15)$ intraspecific hybrids of $T$. krapovickasii and T. scabra and tetraploid $(2 \mathrm{n}=4 \mathrm{x}=20)(2 \mathrm{n}=4 \mathrm{x}=20)$ T. krapovickasii $\times T$. scabra and $T$. subulata $\times T$. scabra hybrids. In the triploid hybrids we found cells with 1 to 5 trivalents and the pollen viability was $11.4 \%$ $15.2 \%$. In the tetraploid hybrids we found cells with 1 to 5 quadrivalents and the pollen viability was $76 \%$ $-87.97 \%$. The results of this work revealed that the reproductive isolation between diploids and tetraploids is incomplete, and that the triploid hybrids are not completely steriles. Likewise, the cytogenetic analysis of the interspecific hybrids evidenced that the genomes of $T$. krapovickasii, T. scabra and T. subulata display more affinity at tetraploid level than at diploid level.
\end{abstract}

Key words: Turnera, meiosis, genomic relationships, triploid, tetraploid.

\begin{abstract}
Resumen: A fin de investigar las relaciones genómicas entre especies de la serie Turnera $(x=5)$, se lleva a cabo un programa de cruzamientos controlados y se obtuvieron numerosos híbridos. Entre las especies con flores amarillas de la serie Turnera, T. krapovickasii, T. scabra y T. subulata poseen citotipos diploide y autotetraploide. Hasta el momento se desconocen el grado de aislamiento reproductivo entre diploides y tetraploides y el grado de afinidad genómica de estas especies a nivel tetraploide. En este trabajo se presentan los resultados del análisis citogenético de los híbridos intraespecíficos triploides $(2 \mathrm{n}=3 \mathrm{x}=15)$ de $T$. krapovickasii y $T$. scabra y los híbridos tetraploides $(2 \mathrm{n}=4 \mathrm{x}=20) T$. krapovickasii $\times T$. scabra y $T$. subulata $\times T$. scabra. En los híbridos triploides se hallaron 1-5 trivalentes y la viabilidad del polen fue $11,3 \%-15,2 \%$. En los híbridos tetraploides se encontraron células con hasta 5 cuadrivalentes y la viabilidad del polen fue $76 \%-87,97 \%$. Los resultados obtenidos demostraron que el aislamiento reproductivo entre diploides y tetraploides es incompleto y que los híbridos triploides no son completamente estériles. Asimismo, se evidenció que los genomios de T. krapovickasii, T. scabra y $T$. subulata serían más afines a nivel tetraploide que a nivel diploide.
\end{abstract}

Palabras Clave: Turnera, meiosis, relaciones genómicas, triploides, tetraploides.

\footnotetext{
${ }^{1}$ Instituto de Botánica del Nordeste (UNNE-CONICET), Casilla de Correo 209, 3400 Corrientes, Argentina.

${ }^{2}$ Facultad de Ciencias Exactas y Naturales y Agrimensura (UNNE), Avenida Libertad 5470, 3400 Corrientes, Argentina.

*Email: silvia.fernandez@comunidad.unne.edu.ar
} 


\section{INTRODUCCIÓN}

La subfamilia Turneroideae comprende 226 especies distribuidas en 12 géneros. De estos géneros, Turnera L. es el más numeroso y cuenta con 141 especies ampliamente distribuidas en las áreas tropicales y subtropicales del continente americano, y dos especies africanas (Arbo et al., 2015). En América, las especies de Turnera se distribuyen desde el sur de los Estados Unidos hasta el centro de Argentina y, algunas especies se han extendido como plantas ruderales a Asia y Oceanía (Arbo, 1995, 2005). Las especies de Turnera se agrupan en once series que se diferencian por la estructura floral, el desarrollo y grado de adnación del pedúnculo floral, el epicarpio, y rasgos seminales como la forma, ornamentación, pubescencia y desarrollo de la cálaza (Arbo, 2008). La heterostilia es también un fenómeno bastante frecuente, las especies pueden ser heterostilas $u$ homostilas, y las heterostilas son generalmente autoincompatibles (Barrett, 1978; Arbo, 1987).

Los estudios cromosómicos mostraron que la poliploidía desempeñó un papel muy importante en la evolución de las especies de Turnera (Fernández, 1987). Se cuenta con información cariológica para 6 de las 11 series (Barrett, 1978; Fernández, 1987; Raman \& Kesavan, 1964; Hamel, 1965; Barrett \& Shore, 1980; Arbo \& Fernández, 1983; Solís Neffa \& Fernández, 1993; Solís Neffa, 1996; Solís Neffa \& Fernández, 2002). El número básico $\mathrm{x}=7$ es el más frecuente $\mathrm{y}$ fue hallado en las series Salicifoliae, Stenodictyae, Microphyllae $\mathrm{y}$ Leiocarpae. El número $\mathrm{x}=13$ fue hallado en la serie Papilliferae y $\mathrm{x}=5$ en la serie Turnera. Se detectaron niveles de ploidía desde $2 \mathrm{x}$ hasta 10x (Barrett, 1978; Fernández, 1987; Raman \& Kesavan, 1964; Arbo \& Fernández, 1983; Shore \& Barrett, 1985; Solís Neffa \& Fernández, 2001; Elías et al., 2011; Kovalsky \& Solís Neffa, 2012). Los estudios meióticos en poliploides indican que hay alo- y autopoliploides (Fernández, 1987; Shore, 1991; Solís Neffa \& Fernández, 2001).

Actualmente, las investigaciones que se desarrollan en el género Turnera tienen como uno de sus objetivos interpretar los mecanismos involucrados en el origen y establecimiento de los poliploides (Fernández \& Arbo, 1990, 2000 a y b; Fernández \& Solís Neffa, 2004; Panseri et al., 2008; Fernández, et al., 2010; Kovalsky \& Solís
Neffa, 2012, 2015; Kovalsky et al., 2014; Kovalsky \& Solís Neffa, 2015). La serie Turnera cuenta con 22 especies que presentan la estructura floral más compleja en la familia y están divididas en dos subseries por sus caracteres seminales (Arbo, 1986) y citogenéticos (Arbo, 2005). La subserie Umbilicatae comprende algunas especies tropicales, mientras que la subserie Turnera constituye el complejo T. ulmifolia. Este último es un complejo polimórfico originariamente constituido por más de 10 variedades (Urban, 1883) que poseen flores amarillas y blanco - azuladas, muchas de las cuales son reconocidas actualmente como especies independientes (Backer, 1951; Arbo, 1985). Desde 1982, se lleva a cabo un programa de cruzamientos controlados entre las especies de la serie Turnera (principalmente de la subserie Turnera) y se han obtenido numerosos híbridos. Como resultado de los estudios citogenéticos de dichos híbridos, se han analizado las relaciones genómicas entre algunas especies (Fernández, 1997; Fernández \& Arbo, 1989, 1990, 1993a, 1993b, 1996, 2000a, 2000b; Fernández \& Solís Neffa, 2004; Fernández et al., 2010; López et al. 2010 a, 2010b).

Entre las especies con flores amarillas de la serie Turnera, T. krapovickasii Arbo, T. scabra Millsp. y T. subulata $\mathrm{Sm}$. poseen citotipo diploide $2 \mathrm{n}=$ $2 \mathrm{x}=10$ y autotetraploide $2 \mathrm{n}=4 \mathrm{x}=20$ (Fernández, 1987; Arbo, 2005; Shore et al., 2006). Los citotipos de estas especies están segregados espacialmente, aunque se han detectado algunas poblaciones mixtas (Shore \& Barrett, 1986; Barrett \& Shore, 1987; Lazaroff et al., 2016). En dichas poblaciones, el grado de aislamiento reproductivo entre diploides y tetraploides podría tener un importante papel en la coexistencia entre los citotipos (Harlan \& deWet, 1975; de Wet, 1980; Felber \& Bever, 1997; Ramsey \& Schemske, 1998; Burton \& Husband, 2000). Por otra parte, las tres especies difieren en su distribución geográfica, aunque el área entre $T$. subulata y T. scabra se superpone parcialmente, habiéndose detectado poblaciones con algunos híbridos (Arbo \& Fernández, 1987; Fernández \& Arbo, 1989; Arbo, 2005). A partir del análisis citogenético de los híbridos diploides, se demostró que las barreras reproductivas entre las tres especies mencionadas es incompleto y se sugirió que sus genomas serían muy afines (Fernández \& Arbo, 1989, 1993). Sin embargo, hasta el momento no se cuenta con información sobre el grado 
de aislamiento reproductivo entre diploides y tetraploides ni tampoco acerca del grado de afinidad genómica de estas especies a nivel tetraploide.

En este marco, en este trabajo se presentan los resultados del análisis citogenético de los híbridos triploides de T. krapovickasii, T. scabra y de los híbridos tetraploides entre estas especies y entre $T$. subulata y T. scabra.

\section{Materiales y Métodos}

Se llevó a cabo un programa de cruzamientos bajo condiciones controladas de invernáculo. Las accesiones utilizadas como progenitores en el presente estudio se citan en la Tabla 1, junto a los códigos empleados, los números cromosómicos, coleccionistas, fechas y localidades de colección. Los números cromosómicos de las plantas empleadas como progenitores fueron obtenidos previamente (Fernández, 1987). Los ejemplares testigo están depositados en el herbario del Instituto de Botánica del Nordeste (CTES), Corrientes, Argentina.

Aunque las especies empleadas en los cruzamientos son autoincompatibles, las flores de las plantas utilizadas como progenitores femeninos fueron castradas previamente a la polinización con el polen de la planta seleccionada como progenitor masculino. Las flores polinizadas se marcaron con una etiqueta indicando el número de cruzamiento y los frutos en desarrollo se encerraron en una bolsa de tul de malla fina para asegurar la cosecha de las semillas. Las semillas obtenidas se sembraron en macetas individuales y los individuos resultantes fueron transplantados luego de haberse desarrollado el primer par de hojas.

La cruzabilidad entre los citotipos y especies se estimó a partir de los siguientes parámetros: el número de frutos y semillas obtenidos en relación al número de flores polinizadas, el promedio de semillas viables por fruto y la viabilidad del polen de los híbridos obtenidos.

El nivel de ploidía de la progenie $\mathrm{F}_{1}$ se determinó en meiosis. Para el estudio de la meiosis en algunos casos se utilizaron anteras frescas y en otros los botones florales fueron fijados en 5 partes de etanol absoluto y una parte de ácido láctico (Fernández, 1973). Después de 24 horas fueron transferidos a etanol $70^{\circ}$ en refrigeración a $4^{\circ} \mathrm{C}$. La coloración se efectuó con orceína acética al $2 \%$. Los preparados permanentes se hicieron con el método de Bowen (1956). Las configuraciones meióticas fueron analizadas en diacinesis - metafase I.

La viabilidad del polen de los híbridos fue estimada mediante la técnica de coloración

Tabla 1. Material estudiado de Turnera.

\begin{tabular}{|c|c|c|c|c|}
\hline Especie & Código & $2 n$ & Coleccionista y procedencia & $\begin{array}{l}\text { Fecha de } \\
\text { colección }\end{array}$ \\
\hline \multirow[t]{7}{*}{ T. krapovickasii Arbo } & $\mathrm{K} 1$ & 10 & Ahumada 4549, Argentina, Jujuy, Capital. & $12 / 21 / 1982$ \\
\hline & $\mathrm{K} 2$ & 10 & Krapovickas 38858, Bolivia, Tarija, O’Connor. & $4 / 29 / 1983$ \\
\hline & K3 & 10 & Krapovickas 39177, Bolivia, Tarija, Gran Chaco. & $5 / 5 / 1983$ \\
\hline & K4 & 10 & Krapovickas 39099, Bolivia, Tarija, O’Connor. & $5 / 2 / 1983$ \\
\hline & K4as & 10 & Arbo 2706, androestéril de K4 cultivada en Corrientes. & - \\
\hline & K5 & 20 & Schinini 19514, Argentina, Salta, Anta. & $12 / 8 / 1979$ \\
\hline & K6 & 20 & Beck 9433, Paraguay, Nueva Asunción, Gral. Garay. & $10 / 5 / 1983$ \\
\hline \multirow[t]{4}{*}{ T. scabra Millsp. } & 12 & 10 & Araquistain 1354, Nicaragua, Managua, Península de Chiltepe. & 2/18/1980 \\
\hline & 14 & 10 & Montiel s/n, Nicaragua, Manágua. & 03/1986 \\
\hline & 11 & 20 & Jiménez 8769, Rep. Dominicana, La Vega, Loma del Puerto. & $3 / 16 / 1980$ \\
\hline & 16 & 20 & Vodicka 879, Haití, Carrefour. & $8 / 26 / 1985$ \\
\hline T. subulata Smith & E4 & 20 & Arbo 2408, Brasil, Piauí, Teresina. & $1 / 28 / 1981$ \\
\hline
\end{tabular}


con carmín-glicerina (1:1). Las anteras fueron colectadas durante las primeras horas de la mañana y los granos de polen fueron extraídos en una gota de colorante carmín-glicerina. Los granos de polen teñidos totalmente se consideraron como viables y los traslúcidos o con poca coloración como inviables. Se contaron al menos 300 granos por planta.

\section{Resultados}

Los resultados obtenidos se resumen en la Figura $1 \mathrm{y}$ en las Tablas 2, 3 y 4.

De los 112 cruzamientos realizados, el $39 \%$ fue exitoso (Tabla 2). En general, los cruzamientos $2 x$ $\times 4 \mathrm{x}$ y $4 \mathrm{x} \times 2 \mathrm{x}$ realizados en $T$. krapovickasii y en T. scabra, fueron exitosos, excepto el cruzamiento I2 $(2 \mathrm{x}) \times \mathrm{I} 1(4 \mathrm{x})$ en el cual ninguna de las flores polinizadas produjo frutos (Tabla 2 ).

En cuanto a las distintas variables estimadas para cada tipo de cruzamiento, en T. krapovickasii, el mayor porcentaje de frutos se obtuvo del cruzamiento K1 $(2 \mathrm{x}) \times \mathrm{K} 5(4 \mathrm{x})$ y el menor porcentaje del cruzamiento K4as $(2 \mathrm{x}) \times \mathrm{K} 5(4 \mathrm{x})$; mientras que el mayor promedio de semillas por fruto se obtuvo de los cruzamientos $2 \mathrm{x} \times$ 4x. En T. scabra, sólo resultaron exitosos los cruzamientos $4 \mathrm{x} \times 2 \mathrm{x}$. Por otra parte, en los cruzamientos interespecíficos entre los tetraploides de $T$. krapovickasii y T. scabra, todas las variables analizadas fueron mayores cuando $T$. krapovickasii fue empleada como progenitor femenino. En el caso del cruzamiento entre $T$. subulata y $T$. scabra, si bien solo dos cruzamientos fueron exitosos, ambos resultaron en un alto número de semillas.

En relación al comportamiento meiótico, en la Tabla 3 se muestran el promedio de las asociaciones cromosómicas y la fertilidad de polen de los híbridos intercitotipos e interespecíficos obtenidos. Los triploides de ambas especies analizadas presentaron diferentes configuraciones en diacinesis y MI, en las que se encontraron 1-5 III. En T. krapovickasii la configuración más frecuente fue $3 \mathrm{I}+3 \mathrm{II}+2$ III (41,66 \%, Fig. 1.A, Tabla 4). En este híbrido se encontró una célula con $1 \mathrm{II}+1 \mathrm{III}+1 \mathrm{X}$ (Fig. 1.B), la cual no fue incluida en la Tabla 3. En el triploide de $T$. scabra se encontraron seis configuraciones diferentes, siendo las más frecuentes $1 \mathrm{I}+1 \mathrm{II}+4$ III (39,33\%, Fig. 1.C) y 5 III $(25,84 \%$, Fig. $1 . D)$. En los híbridos triploides de ambas especies siempre se encontró al menos un trivalente por célula y también se observaron cromosomas rezagados y puentes en anafase I y en anafase II. La fertilidad de polen de los triploides varió entre $11,30 \%$ y 15,20 $\%$ (Tabla 3).

En los híbridos tetraploides T. krapovickasii $\times T$. scabra y T. subulata $\times T$. scabra, la configuración más frecuente fue de $2 \mathrm{II}+4 \mathrm{IV}$, con un $33 \%$ en el primer híbrido y 44,45\% en el segundo (Tabla 4). Se encontraron células con univalentes, bivalentes y cuadrivalentes en el primer híbrido, mientras que en el segundo además de estas asociaciones también se observaron trivalentes. En todas las células analizadas de T. krapovickasii $\times$ T. scabra se encontraron cuadrivalentes, hallándose un máximo de 5 IV (Fig. 1. F) en el $14 \%$ de las células analizadas. La viabilidad del polen fue alta con un 87,97\% (Tabla 3) en T. krapovickasii $\times$ T. scabra mientras que en T. subulata $\times$ T. scabra fue de 76 $\%$. En anafase I se encontró $6,5 \%$ de cromosomas rezagados y $8 \%$ de puentes en $T$. krapovickasii $\times$ T. scabra, en tanto que en T. subulata $\times T$. scabra se encontraron valores similares con $7 \%$ de cromosomas rezagados y 10,7 \% de puentes. En el primer híbrido se observaron solamente puentes, mientras que en el segundo se encontraron puentes $\mathrm{y}$ fragmentos.

\section{Discusión}

Los resultados obtenidos en este trabajo demostraron que el aislamiento reproductivo entre los citotipos diploides y tetraploides de $T$. krapovickasii y $T$. scabra no es absoluto y que los triploides resultantes de los cruzamientos experimentales no son completamente estériles. Asimismo, el análisis citogenético de los híbridos interespecíficos tetraploides evidenció que los genomios de T. krapovickasii, T. scabra y $T$. subulata serían muy afines entre sí.

Híbridos triploides. En algunos complejos poliploides, los tetraploides con frecuencia están aislados reproductivamente de sus progenitores diploides por fuertes barreras postcigóticas, las que involucran mecanismos que conducen a la inviabilidad de los híbridos (bloqueo triploide) y a la esterilidad (Ramsey \& Schemske, 1998; Futuyma, 1998; Petit et al., 1999; Schuter, 2001). Los 


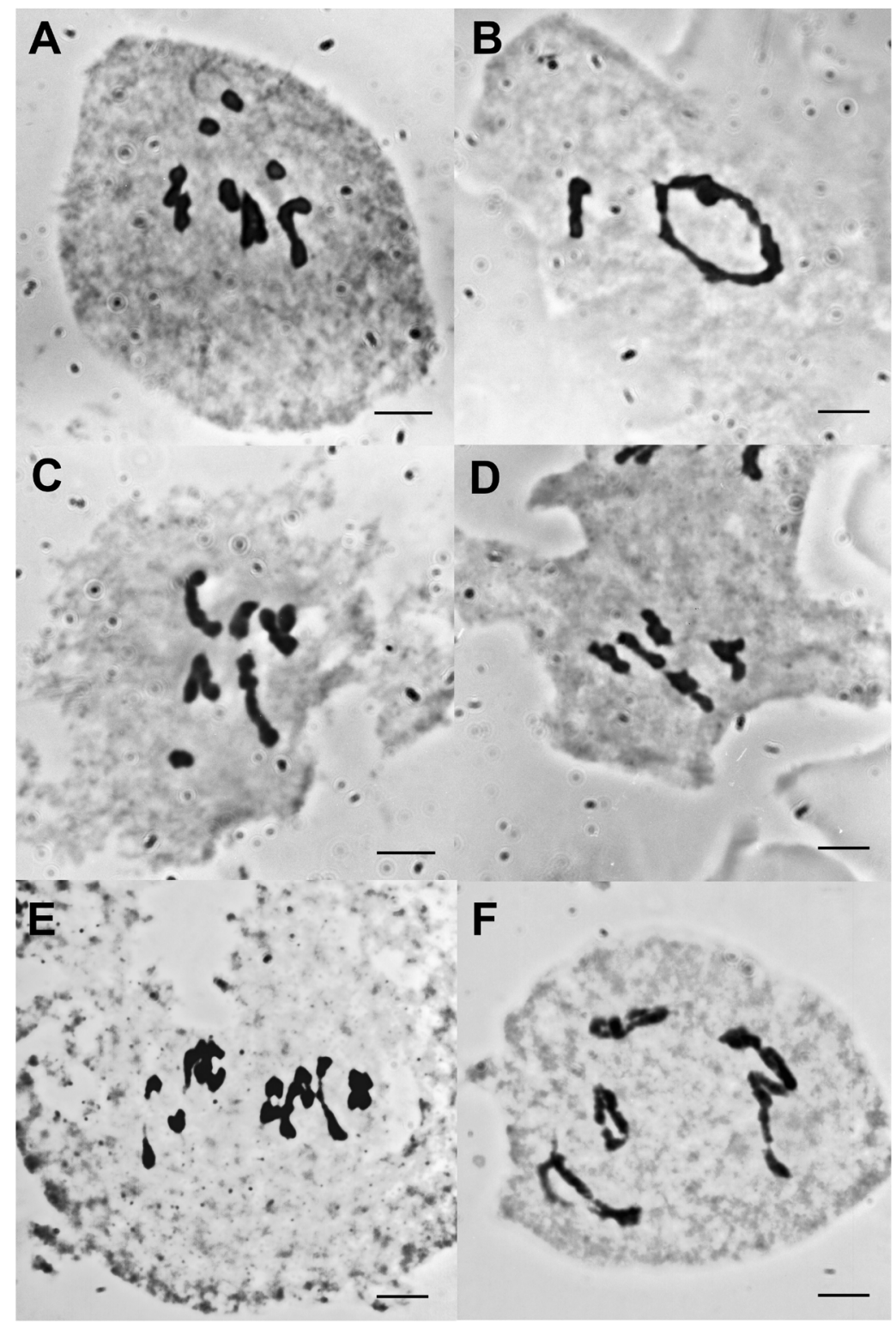

Fig. 1. A-B: T. krapovickasii, $2 \mathrm{n}=3 \mathrm{x}=15 . \mathrm{A}: 3 \mathrm{I}+3 \mathrm{II}+2 \mathrm{III}$. B: $1 \mathrm{II}+1 \mathrm{III}+1 \mathrm{X}$. C-D: $T$. scabra, $2 \mathrm{n}=3 \mathrm{x}=15$. C: 1 I + 1 II + 4 III. D: 5 III. E-F: T. krapovickasii $\times$ T. scabra, $2 n=4 x=20 . E: 4 I I+3$ IV. F: 5 IV. Escala: $5 \mu \mathrm{m}$.

embriones triploides abortan en etapas tempranas del desarrollo como resultado del desbalance de la relación del nivel de ploidía del embrión y del endosperma o de la proporción materno: paterna (bloqueo triploide) (Ramsey \& Schemske, 1998; Köhler et al., 2010). El bloqueo triploide varía según las especies (Marks, 1966), pudiendo ser fuerte como en Solanum (Werner \& Peloquín, 1991a), Trifolium pratense L. (Taylor \& Wieseman, 1988) o en Lotus tenuis Wald. et Kit. (Negri \& Veronessi, 1989). En estas especies, los triploides son raros o pueden estar ausentes (Lumaret et al. 1987; van Dijk et al., 1992). Sin embargo, la presencia de triploides en las zonas de contacto entre diploides y tetraploides así como en la progenie de cruzamientos experimentales en otras 
Bol. Soc. Argent. Bot. 52 (4) 2017

Tabla 2. Resultados de los cruzamientos experimentales.

\begin{tabular}{|c|c|c|c|c|}
\hline Cruzamiento & $\begin{array}{c}\text { Número de flores } \\
\text { polinizadas }\end{array}$ & $\begin{array}{l}\text { Número de frutos } \\
\text { producidos }\end{array}$ & $\begin{array}{l}\text { Número promedio de } \\
\text { semillas por fruto }\end{array}$ & $\begin{array}{l}\text { Nivel de ploidía } \\
\text { de la progenie }\end{array}$ \\
\hline $\mathrm{K} 1(2 \mathrm{x}) \times \mathrm{K} 5(4 \mathrm{x})$ & 21 & $13(61,90 \%)$ & $\begin{array}{c}6.8 \\
(1-17)\end{array}$ & $3 x$ \\
\hline $\mathrm{K} 4$ as $(2 \mathrm{x}) \times \mathrm{K} 6(4 \mathrm{x})$ & 11 & $3(27,27 \%)$ & $\begin{array}{c}2.67 \\
(1-6)\end{array}$ & $3 x$ \\
\hline $\begin{array}{c}\mathrm{K} 5(4 \mathrm{x}) \times \mathrm{K} 1(2 \mathrm{x}) \\
\mathrm{I} 2(2 \mathrm{x}) \times \mathrm{I}(4 \mathrm{x})\end{array}$ & $\begin{array}{l}12 \\
20\end{array}$ & $\begin{array}{c}5(41,67 \%) \\
0(0,00 \%)\end{array}$ & $\begin{array}{c}1.4 \\
(1-3) \\
-\end{array}$ & $\begin{array}{l}3 x \\
-\end{array}$ \\
\hline $11(4 x) \times 12(2 x)$ & 26 & $12(46,15 \%)$ & $\begin{array}{c}3.11 \\
(1-10)\end{array}$ & $3 x$ \\
\hline$K 5(4 x) \times I 1(4 x)$ & 3 & $3(100 \%)$ & $\begin{array}{c}17 \\
(1-41)\end{array}$ & $4 x$ \\
\hline $\mathrm{I} 1(4 \mathrm{x}) \times \mathrm{K} 5(4 \mathrm{x})$ & 7 & $6(85,71 \%)$ & $\begin{array}{c}13.5 \\
(1-22)\end{array}$ & $4 x$ \\
\hline$E 4(4 x) \times I 1(4 x)$ & 12 & $2(16,67 \%)$ & $\begin{array}{c}34 \\
(34) \\
\end{array}$ & $4 x$ \\
\hline
\end{tabular}

especies (Zohary \& Nur, 1959; Felber \& Bever, 1997), evidencian que el bloqueo triploide nunca es absoluto y que puede ser sobrellevado con cierta frecuencia. En estas circunstancias, los triploides pueden aparecer con mayor frecuencia (Husband \& Schemske, 1998; Husband, 2004).

En las especies de Turnera con citotipos diploide y autotetraploide, la mayoría de las poblaciones están constituidas por un solo citotipo, aunque también se encontraron poblaciones mixtas diploide - triploide - tetraploide (Elías et al., 2011; Kovalsky \& Solís Neffa, 2012). En T. krapovickasii y $T$. scabra, los diploides y tetraploides están segregados espacialmente (Shore \& Barrett, 1986; Barrett \& Shore, 1987; Lazaroff et al., 2016), aunque también se detectaron algunos individuos triploides en las poblaciones naturales diploides de T. krapovickasii (Lazaroff et al., 2016). Dicho hallazgo, sumado a la presencia de triploides en la progenie de los cruzamientos experimentales entre diploides y tetraploides de $T$. krapovickasii y $T$. subulata detectados en este trabajo, evidencian que el bloqueo triploide también puede ser superado en estas especies.

Asimismo, se ha sugerido que el aislamiento reproductivo entre los citotipos puede ser asimétrico (Husband \& Sabara, 2003). El colapso de los embriones debido a desbalances en el nivel de ploidía o en la relación materno : paterna en el embrión / endosperma puede ser sobrellevado

Tabla 3. Promedio \pm ES y variaciones de las asociaciones cromosómicas en MI de los de los híbridos triploides y tetraploides de T. krapovickasii, T. scabra y T. subulata.

\begin{tabular}{|c|c|c|c|c|c|c|c|}
\hline Híbrido & $\begin{array}{l}\text { Nivel de } \\
\text { ploidía }\end{array}$ & I & II & III & IV & CMP & $\begin{array}{c}\text { Viabilidad del } \\
\text { polen (\%) }\end{array}$ \\
\hline \multirow[t]{2}{*}{$11 \times 12$} & $3 x$ & $1,19 \pm 0,10$ & $1,15 \pm 0,09$ & $3,83 \pm 0,09$ & - & 22 & 15.2 \\
\hline & & $(0-4)$ & $(0-3)$ & $(2-5)$ & - & & \\
\hline \multirow[t]{2}{*}{ K4as x K6 } & $3 x$ & $2,08 \pm 0,37$ & $2,03 \pm 0,37$ & $2,91 \pm 0,37$ & - & 89 & 11.3 \\
\hline & & $(0-4)$ & $(0-4)$ & $(1-5)$ & - & & \\
\hline \multirow[t]{2}{*}{$\mathrm{K} 5 \times \mathrm{I} 1$} & $4 x$ & $0,09 \pm 0,09$ & $3,57 \pm 0,53$ & - & $3,19 \pm 0,27$ & 21 & 87.97 \\
\hline & & $(0-2)$ & $(0-8)$ & - & $(1-5)$ & & \\
\hline \multirow[t]{2}{*}{$\mathrm{E} 4 \times \mathrm{I1}$} & $4 x$ & $0,22 \pm 0,14$ & $3,55 \pm 0,72$ & $0,22 \pm 0,14$ & $3 \pm 0,37$ & 12 & 76 \\
\hline & & $(0-1)$ & $(0-8)$ & $(0-1)$ & $(1-5)$ & & \\
\hline
\end{tabular}


A. Fernández et al. - Híbridos triploides y tetraploides en Turnera

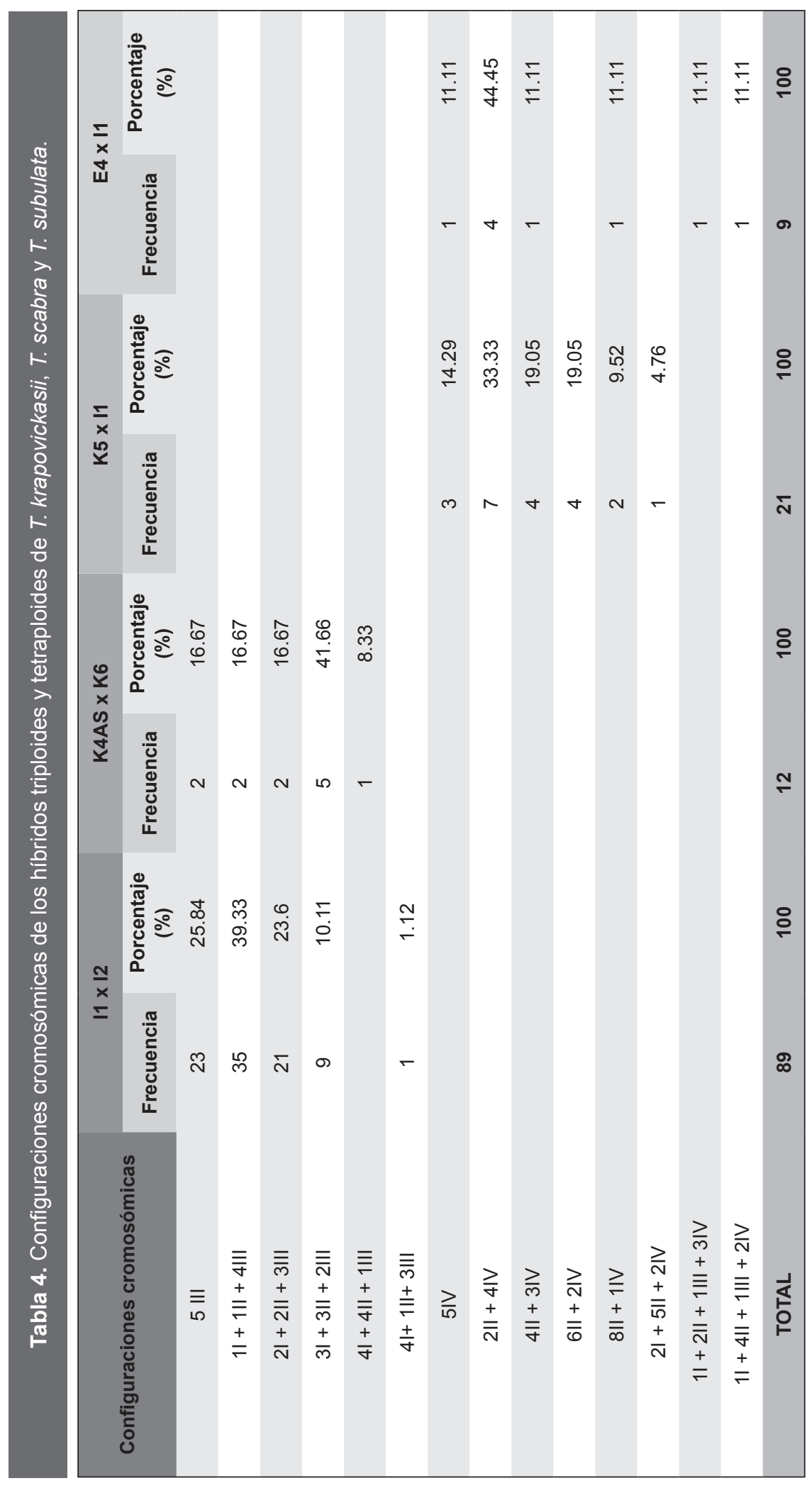


cuando la planta con el nivel de ploidía más alto es empleada como progenitor femenino, como fuera observado en algunos cruzamientos interploidía involucrando otras especies de Turnera (Shore \& Barret, 1985; Arbo \& Fernández, 1987, Fernández \& Solís Neffa, 2004; Fernández et al., 2010) así como especies de otros géneros (Woodell \& Valentine, 1961, Ockendon, 1968; Levin, 1971). Los resultados obtenidos en este trabajo sugieren que la asimetría en los cruzamientos interploidía podría variar entre las especies de Turnera, ya que aunque en $T$. scabra los cruzamientos sólo fueron exitosos cuando los tetraploides fueron utilizados como progenitores femeninos, en T. krapovickasii el número de cruzamientos exitosos, así como de frutos y semillas obtenidos, fue variable en las diferentes combinaciones empleadas en los cruzamientos.

Por otra parte, la eficacia reproductiva de los triploides y el tipo de gametos $(n=1 x, n=2 x$ y $n=$ $3 x$ ) que forman pueden contribuir a la dinámica de las zonas de contacto entre citotipos (Harlan \& deWet, 1975; de Wet, 1980; Felber \& Bever, 1997; Ramsey \& Schemske, 1998; Burton \& Husband, 2000). En las zonas de contacto entre citotipos, la producción de triploides estériles, como resultado de las hibridaciones entre diploides y tetraploides, puede favorecer la evolución de barreras reproductivas entre los dos citotipos y, de este modo, contribuir a la segregación espacial de ambas poblaciones. Por el contrario, aún con una fertilidad parcial, los triploides pueden actuar como intermediarios en la producción de tetraploides (hipótesis del triploide - puente) (Harlan \& de Wet, 1975; de Wet, 1980) y/o contribuir al flujo génico entre diploides y tetraploides (Stebbins, 1971; Levin, 1975; Felber \& Bever, 1997; Lenormand, 2002; Pannell et al., 2004; Stift et al., 2010).

Los resultados aquí obtenidos demostraron que los triploides de T. krapovickasii y T. scabra, no son completamente estériles. Los porcentajes de viabilidad del polen de dichos triploides concuerdan con los detectados previamente en triploides provenientes de poblaciones naturales y en los resultantes de cruzamientos experimentales (Arbo \& Fernández, 1983; Elías, 2009; Kovalsky, 2012). Las causas de la infertilidad de los poliploides son complejas e incluyen tanto aberraciones meióticas, como los efectos fisiológicos y los factores génicos de la poliploidía (Comai, 2005). En particular, en los triploides, la ocurrencia de univalentes y trivalentes sería la principal causa de esterilidad, ya que la presencia de los mismos complica la segregación meiótica normal de los cromosomas homólogos, los que se dividen desigualmente durante anafase I (Sybenga, 1975). La alta frecuencia de univalentes y trivalentes detectada en los triploides de $T$. krapovickasii y T. scabra sería, por lo tanto, la causa de la reducción de la viabilidad del polen de los triploides. Por otra parte, la detección de polen viable en los triploides de T. krapovickasii y T. scabra sugiere que dichos triploides forman gametos viables (aunque en baja proporción), los que podrían intervenir en la formación de neopoliploides mediante poliploidización sexual unilateral, como se comprobó en T. sidoides (Kovalsky \& Solís Neffa, 2012, 2015).

Asimismo, el mayor número promedio de trivalentes que de univalentes y bivalentes detectado en los híbridos triploides de T. krapovickasii y $T$. scabra, evidencia la continuidad genética entre diploides y tetraploides. Este hecho sumado a que los triploides de T. krapovickasii y T. scabra no son totalmente estériles, sugiere que dichos triploides podrían tener un importante papel en el flujo génico entre citotipos en las poblaciones mixtas o en las zonas de contacto diploide-tetraploide.

Es interesante señalar que en el híbrido triploide de $T$. krapovickasii $\mathrm{K} 4 \mathrm{as} \times \mathrm{K} 6$ se encontró una célula con una asociación cromosómica de decavalente, la única explicación posible es que se deba a una translocación múltiple entre los cromosomas de las dos accesiones (K4as y K6). Probablemente esta translocación múltiple involucre segmentos muy pequeños, motivo por el cual se encontró una sola célula con esta asociación.

Híbridos tetraploides. T. krapovickasii y T. scabra están aisladas geográficamente. $T$. krapovickasii vive en el norte Argentino y sur de Bolivia, mientras que T. scabra crece en el norte de Brasil y Centro América. Los resultados del análisis citogenético tanto de los híbridos tetraploides aquí obtenidos como de los híbridos diploides obtenidos previamente (Fernández \& Arbo, 1989) sugieren que, a pesar del aislamiento geográfico, el aislamiento reproductivo entre ambas especies es incompleto.

Los resultados del análisis citogenético de los híbridos diploides obtenidos previamente (Fernández \& Arbo, 1989) y de los híbridos 
tetraploides de T. krapovickasii $\times$ T. scabra y $T$. subulata $\times T$. scabra obtenidos en este trabajo, sugieren que la capacidad de cruzamiento y las barreras de esterilidad entre las especies están más desarrolladas a nivel diploide; mientras que los tetraploides se cruzan con bastante facilidad entre sí para producir híbridos con un alto grado de fertilidad. Además, el porcentaje de viabilidad del polen es menor en los híbridos diploides $(35,7 \%$ - 69,85\%, en Fernández \& Arbo, 1989) que en los híbridos tetraploides $(76 \%-87,97 \%)$. En los híbridos diploides en metafase I se encontraron células con tres a cinco bivalentes (Fernández \& Arbo, 1989); mientras que los híbridos tetraploides aquí estudiados presentaron cuadrivalentes en todas las células (hasta el máximo posible de 5 cuadrivalentes). De acuerdo con estos resultados, T. krapovickasii, T. scabra y T. subulata serían más afines a nivel tetraploide que a nivel diploide.

T. subulata y T. scabra conviven en Colombia, Venezuela y Brasil (Arbo, 2005), hibridan con facilidad (Arbo \& Fernández, 1987; Fernández \& Arbo, 1989; Shore \& Barrett, 1985) y sus cariotipos son altamente semejantes (Solís Neffa \& Fernández, 1993). Los análisis citogenéticos mediante técnicas clásicas y moleculares indican que existe una homología considerable entre los genomios de T. scabra, T. subulata y $T$. krapovickasii (López et al. 2013). De acuerdo a Fernández y Arbo (1989) probablemente $T$. subulata haya dado origen a $T$. scabra y $T$. krapovickasii, esta deducción surgió a partir de la interpretación de la distribución geográfica y del análisis citogenético de los híbridos diploides entre estas especies. Los datos de cruzabilidad, comportamiento meiótico y viabilidad de polen de los híbridos tetraploides obtenidos en este trabajo sustentan esta hipótesis.

\section{Agradecimientos}

Este trabajo fue financiado por proyectos de la Secretaría General de Ciencia y Técnica (P 004/14) y del Consejo Nacional de Investigaciones Científicas y Técnicas (CONICET PIP 11220120100192CO). S. A. Fernández es becaria doctoral CONICET. I. E. Kovalsky es becaria postdoctoral ANPCyTFONCyT. V. G. Solís Neffa es miembro de la Carrera del Investigador Científico de CONICET.

\section{Bibliografía}

ARBO, M. M. 1985. Notas taxonómicas sobre Turneráceas sudamericanas. Candollea 40: 175-191.

ARBO, M. M. 1986. Paraguay, centro importante de especiación en las Turneráceas. Candollea 41:211218.

ARBO, M. M. 1987. Turneraceae. En: SPICHIGER R, (ed.). Flora del Paraguay Conservatoire et Jardin Botaniques de Genève \& Missouri Botanical Garden, 6: 1-65.

ARBO, M. M. 1995. Turneraceae Parte I. Piriqueta. Flora Neotropica Monog. 67: 1-156.

ARBO, M. M. 2005. Estudios sistemáticos en Turnera (Turneraceae) III. Series Anomalae y Turnera. Bonplandia 14(3-4): 115-318.

ARBO, M. M. 2008. Estudios sistemáticos en Turnera (Turneraceae) IV. Series Leiocarpae, Sessilifoliae y Conciliatae. Bonplandia 17:107-334.

ARBO, M. M. \& A. FERNÁNDEZ. 1983. Posición taxonómica, citología y palinología de tres niveles de ploidía de Turnera subulata Smith. Bonplandia 5: 111-226.

ARBO, M. M. \& A. FERNÁNDEZ. 1987. Cruzamientos intra e interespecíficos en Turnera, serie Canaligerae. Bonplandia 6(1): $23-28$.

ARBO, M. M., A. GONZÁLEZ \& S. SEDE. 2015. Phylogenetic relationships within Turneraceae based on morphological characters with emphasis on seed micromorphology. Plant. Syst. Evol. 301 (7): $1907-$ 1926.

BACKER, C. A. 1951. Turneraceae. Flora Malesiana Ser. 1, 4: 235-238.

BARRETT, S. C. H. 1978. Heterostyly in a tropical weed: the reproductive biology of the Turnera ulmifolia complex (Turneraceae). Can. J. Bot. 56(15): 17131725.

BARRETT, S. C. H. \& J. S. SHORE. 1980. Variation in breeding systems in the Turnera ulmifolia complex. Trabajo presentado en el Second International Congress of Systematic and Evolutionary Biology, Vancouver, Canadá.

BARRETT, S. C. H. \& J. S. SHORE. 1987. Variation and evolution of breeding systems in the Turnera ulmifolia L. complex (Turnearaceae). Evolution 41: 340-354.

BOWEN, C. C. 1956. Freezing by liquid carbone dioxide in making slides permanent. Stain Technol. 31: 8790.

BURTON, T. L. \& B. C. HUSBAND. 2000. Fitness differences among diploids and tetraploids and their triploid progeny in Chamerion angustifolium (Onagraceae): mechanisms of inviability and implications for polyploid evolution. Evolution 54:1182-1191. 
COMAI, L. 2005. The advantages and disadvantages of being polyploid. Nat. Rev. Genet. 6: 836-846.

DE WET, J. M. J. 1980. Origins of polyploids. — In: Lewis, W. H. (ed.), Polyploidy, biological relevance: 3-16. Plenum Press, New York.

ELÍAS, G. 2009. Dinámica de una zona de contacto diploide-tetraploide de Turnera sidoides subsp. Pinnatifida (Turneraceae). Tesis Doctoral. Universidad Nacional de-Tucumán.

ELÍAS, G.; M. SARTOR \& V. G. SOLÍS NEFFA. 2011. Patterns of cytotype variation of Turnera sidoides subsp. pinnatifida (Turneraceae) in mountain ranges of central Argentina. J. Plant. Res. 124: 25-34.

FELBER, F. \& J. D. BEVER. 1997. Effect of triploid fitness on the coexistence of diploids and tetraploids. Biol. J. Linn. Soc. 60: 95-106.

FERNÁNDEZ, A. 1973. El ácido láctico como fijador cromosómico. Bol. Soc. Argen. Bot. 15(2-3):287-290.

FERNÁNDEZ, A. 1987. Estudios cromosómicos en Turnera y Piriqueta (Turneraceae). Bonplandia 6(1): $1-21$.

FERNÁNDEZ, A. 1997. Estudio citogenético en híbridos entre una especie octoploide, Turnera aurelii y dos diploides, T. caerulea y T. joelii. Bonplandia 9:281286.

FERNÁNDEZ, A. \& M. M. ARBO. 1989. Relaciones genómicas entre cuatro especies diploides de Turnera con flores amarillas (Serie Canaligerae). Bonplandia 6(2): 93-109.

FERNÁNDEZ, A. \& M. M. ARBO. 1990. Gametas no reducidas y relaciones genómicas en tres especies de Turnera (Turneraceae). Darwiniana 30: 21 - 26.

FERNÁNDEZ, A. \& M. M. ARBO. 1993a. Relaciones genómicas entre seis especies de Turnera (Serie Canaligarae) del Paraguay. Candollea 48: 305-318.

FERNÁNDEZ, A. \& M. M. ARBO. 1993b. Citogenética de híbridos entre Turnera grandidentata (4x) y T. subulata y T. scabra (Turneraceae). Bonplandia 7:119-127.

FERNÁNDEZ, A. \& M. M. ARBO. 1996. Relaciones genómicas entre las especies diploides de flores blancoazuladas de Turnera (Serie Canaligerae). Bonplandia 9: 95-102.

FERNÁNDEZ, A. \& M. M. ARBO. 2000a. Cytogenetic relationships between Turnera aurelii, T. cuneiformis $(2 \mathrm{n}=8 \mathrm{x}=40)$ and $T$. orientalis $(2 \mathrm{n}=6 \mathrm{x}=30)$ (Turneraceae). Cytologia 65: 97-102.

FERNÁNDEZ, A. \& M. M. ARBO. 2000b. Relaciones genómicas entre dos especies hexaploides de Turnera, T. orientalis y $T$. velutina, y una especie diploide, $T$. grandiflora (Turneraceae, serie Turnera). Bonplandia 10:181-187.

FERNÁNDEZ, A. \& V. G. SOLÍS NEFFA. 2004. Genomic relationships between Turnera krapovickasii (2x, 4x) and T. ulmifolia (6x) (Turneraceae, Turnera). Caryologia 57: 45-51.
FERNÁNDEZ, A., H. REY, \& V.G. SOLÍS NEFFA. 2010. Evolutionary relationships between the diploid Turnera grandiflora and the octoploid T. fernandezii (Serie Turnera, Turneraceae). Ann. Bot. Fenn. 47: 321-329.

FUTUYMA, D. J. 1998. Evolutionary biology. $3^{\text {rd }}$. Sunderland. MA: Sinauer Assoc. Inc.

HAMEL, J. L. 1965. Le noyau et les chromosomes somatiques de Turnera ulmifolia L. Mém. Mus. Natl. His. Nat. Sér. B, Bot. 16(1): 3-8.

HARLAN, J. R. \& J. M. J. DEWET. 1975. En Ö. Winge and a prayer: the origins of polyploidy. Bot. Rev. 41: 361-390.

HUSBAND, B. C. \& D. W. SCHEMSKE. 1998. Cytotype distribution at a diploid - tetraploid contact zone in Chamerion (Epilobium) angustifolium (Onagraceae). Am. J. Bot. 85 (12):1688-1694.

HUSBAND, B. C. \& H. A. SABARA. 2003. Reproductive isolation between autotreploids and their diploid progenitors in fireweed, Chamerion angustifolium (Onagraceae). New Phytol. 161: 703-713.

HUSBAND, B. C. 2004. The role of triploid hybrids in the evolutionay dynamics of mixed-ploidy populations. Biol. J. Linn. Soc. 82: 537-546.

KÖHLER, C., O. MITTELSTEN SCHEID \& A. ERILOVA. 2010. The impact of the triploid block on the origin and evolution of polyploid plants. Trends Genet. 26: 142-148.

KOVALSKY, I. E. 2012. Origen y establecimiento de neopoliploides en poblaciones naturales de Turnera sidoides L. (Turneraceae). Tesis Doctoral. Universidad Nacional de Córdoba, Argentina.

KOVALSKY, I. E. \& V. G. SOLÍS NEFFA. 2012. Evidence of $2 \mathrm{n}$ microspore production in a natural diploid population of Turnera sidoides subsp. carnea and its relevance in the evolution of the $T$. sidoides (Turneraceae) autopolyploid complex. J. Pl. Res. 125: 725-734.

KOVALSKY, I. E. \& V. G. SOLÍS NEFFA. 2015. Análisis de la progenie de individuos productores y no productores de gametos masculinos no reducidos de Turnera sidoides L. (Passifloraceae). Bol. Soc. Argent. Bot. 50:23-33.

KOVALSKY, I. E., A. FERNÁNDEZ \& V. G. SOLÍS NEFFA. 2014. Mecanismos citológicos involucrados en la producción de gametos masculinos no reducidos en individuos diploides de Turnera sidoides subsp. carnea (Passifloraceae). Bol. Soc. Argent. Bot. 49:227-234.

LAZAROFF, Y., E. M. S. MORENO, A. FERNÁNDEZ \& V. G. SOLÍS NEFFA. 2016. Análisis citogeográfico en Turnera krapovickasii (Passifloraceae). Bol. Soc. Argent. Bot. 51 (1): 153-167.

LENORMAND, T. 2002. Gene flow and the limits to natural selection. Trends Ecol. Evol. 17: 183-189. 


\section{A. Fernández et al. - Híbridos triploides y tetraploides en Turnera}

LEVIN, D. A. 1971. The origin of reproductive isolating mechanisms in flowering plants. Taxon 20:91-113.

LEVIN, D. A. 1975. Minority cytotype exclusion in local plants populations. Taxon 24: 35-43.

LOPEZ, A., A. FERNÁNDEZ \& L. POGGIO. 2010a. Genomic affinities in Turnera (subseries Turnera, Turneraceae) inferred by in situ hybridization techniques. Genome 53: 594-598.

LOPEZ, A., L. POGGIO \& A. FERNÁNDEZ. $2010 \mathrm{~b}$. Genomic relationships between Turnera orientalis and T. occidentalis ( Turneraceae). Ann. Bot. Fennici 47: 471-476.

LÓPEZ, A., A. FERNÁNDEZ \& J. S. SHORE. 2013. Inferences on the origins of polyploid Turnera species (Passifloraceae). Botany 91: $167-175$.

LUMARET R., J. L. Guillerm, J. Delay, A. A. L. Loutfi, J. Izco \& M. Jay. 1987. Polyploidy and habitat differentiation in Dactylis glomerata L. from Galicia (Spain). Oecologia 73:436-446.

MARKS, G. E. 1966. The origin and significance of intraspecific polyploidy: experimental evidence from Solanum chacoense. Evolution 20: 552-5.

NEGRI, V. \& F. VERONESSI. 1989. Evidence for the existence of $2 n$ gametes in Lotus tenuis Wald. et Kit $(2 \mathrm{n}=2 \mathrm{x}=12)$; their relevance in evolution of breeding of Lotus corniculatus L. $(2 \mathrm{n}=4 \mathrm{x}=24)$. Theor. Appl. Genet. 78: 400-404.

OCKENDON, D. J. 1968. Biosystematic studies in the Linum perenne group. New Phytol. 67: $787-813$.

PANNELL J. R., D. J. OBBARD \& R. J. BUGGS. 2004. Polyploidy and the sexual system: what can we learn from Mercurialis annua? Biol. J. Linn. Soc. 2: 547-560.

PANSERI A. F., J. G. SEIJO \& V. G. SOLIS NEFFA. 2008. Análisis de la producción y frecuencia de microsporas no reducidas en diploides de Turnera sidoides (Turneraceae). Bol. Soc. Argent. Bot. 43: 95-101.

PETIT, C., F. BRETAGNOLLE \& F. FELBER. 1999. Evolutionary consequences of diploid-polyploid hybrid zones in wild species. Trends Ecol. Evol. 14: 306-311.

RAMAN, V. S. \& P. C. KESAVAN. 1964. Meiosis and nature of polyploidy in Turnera ulmifolia. J. Indian Bot. Soc. 43: 495-499.

RAMSEY, J. \& D. W. SCHEMSKE. 1998. Pathways, mechanisms, and rates of polyploid formation in flowering plants. Annu. Rev. Ecol. Syst. 29: 467-501.

SCHUTER, D. 2001. Ecology and the origin of species. Trends Ecol. Evol. 16: 372-380.

SHORE, J. S. 1991. Tetrasomic inheritance and isozyme variation in Turnera ulmifolia vars. elegans Urb. and intermedia Urb. (Turneraceae). Heredity 66: 305-312.

SHORE, J. \& S. C. H. BARRET. 1985. Morphological differentiation and crossability among populations of the Turnera ulmifolia L. complex (Turneraceae). Syst. Bot. 10(3): 308-321.
SHORE, J. \& S. C. H. BARRETT. 1986. Genetic modifications of dimorphic incompatibility in the Turnera ulmifolia L. complex (Turneraceae). Can. J. Genet. Cytol. 28: 796-807.

SHORE, J. S., M. M. ARBO \& A. FERNÁNDEZ. 2006. Breeding system variation, genetics and evolution in the Turneraceae. New Phytol. 171: 539-551.

SOLÍS NEFFA, V. G. 1996. Cariotipos de especies de Turnera (Turneraceae). Bonplandia 9 (1-2): 121-127.

SOLÍS NEFFA, V. G. \& A. FERNÁNDEZ. 1993. Estudios cromosómicos en especies de Turnera (Turneraceae). Bonplandia 7(1-4): 101- 118.

SOLÍS NEFFA, V. G. \& A. FERNÁNDEZ. 2001. Cytogeography of the Turnera sidoides L. complex (Turneraceae, Leiocarpae). Bot. J. Linn. Soc. 137: 189-196.

SOLÍS NEFFA, V. G. \& A. FERNÁNDEZ. 2002. Karyotypic studies in Turnera sidoides complex (Turneraceae, Leiocarpae). Amer. J. Bot. 89: 551-558.

STEBBINS, G. 1971. Chromosomal evolution in higher plants. Arnold. London, UK.

STIFT M., R. BREGMAN, J. G. B. OOSTERMEIJER \& P. H. VAN TIENDEREN. 2010. Other tetraploid species and conspecific diploids as sources of genetic variation for an autotetraploid. Am. J. Bot. 97: 1858-1866.

SYBENGA, J. 1975. Meiotic Configurations: Meiotic configurations: a source of information for estimating genetic parameters. Springer-Verlag, Berlin.

TAYLOR, N.L. \& E. WIESEMAN. 1988. Triploids and tetraploids from 43-23 crosses in red clover. Crop Sci. 27: 14-18.

URBAN, I. 1883. Monographie der familia der Turneraceen. Jahrb. Konigl. Bot. Gart. 2:1-152.

VAN DIJK, P., M. HARTOG \& W. VAN DELDEN. 1992. Single cytotype areas in autopolyploid Plantago media L. Biol. J. Linn. Soc. 46: 315 - 331.

WOODELL, S. R. J. \& D. H. VALENTINE. 1961: Studies in the British Primulas. IX. Seed incompatibility in diploid- autotetraploid crosses. New Phytol. 60: 282-294.

ZOHARY, D. \& U. NUR. 1959. Natural triploids in the orchard grass, Dactylis glomerata L., polyploid complex and their significance for gene flow from diploid to tetraploid levels. Evolution 13: 311- 17.

Recibido el 21 de diciembre de 2016, aceptado el 9 de febrero de 2017. 
